\title{
Consenso sobre el cuidado del niño con traqueostomía
}

\author{
Clinical consensus statement on the care of the child with a \\ tracheostomy
}

\author{
Dra. Paula Urrestarazu ${ }^{a}$, Dr. Juan Varón ${ }^{a}$, Dra. Aldana Rodríguez ${ }^{a}$, \\ Lic. Valeria Ton ${ }^{a}$, Dr. Fernando Vila ${ }^{b}$, Dra. Silvina Cipriani ${ }^{b}$, Dra. Karina Moncada ${ }^{b}$ \\ Dra. Cintia P. Antoniolib, Lic. María A. Timonic, Lic. Martha E. Altinac, \\ Lic. Yamila B. Nocitic, Lic. Mariana L. Silvac, Lic. Liliana Del Valle Rodríguez, \\ Lic. Esteban Rivas ${ }^{d}$, Dra. Ivanna Boailchuke, Dra. Mary E. Nieto ${ }^{y}$ \\ Dr. Hugo A. Botto $f$
}

a. Grupo de Trabajo en Discapacidad.

b. Comité de Neumonología.

c. Grupo de Trabajo en Kinesiología.

d. Grupo de Trabajo en Enfermería.

Sociedad Argentina

de Pediatría.

e. Servicio de Endoscopía, Hospital de Niños Ricardo Gutiérrez.

f. Servicio de Endoscopía, Hospital Nacional de Pediatría "Prof. Dr. J. P. Garrahan".

\section{Correspondencia: \\ Dra. Paula Urrestarazu: discapacidadsap@ sap.org.ar}

Financiamiento:

Ninguno.

Conflicto de intereses: Ninguno que declarar.

Recibido: 2-9-2015

Aceptado: 9-9-2015

\section{RESUMEN}

El cuidado del niño con traqueostomía merece especial atención por el potencial riesgo para la vida que podría ocasionarse con el compromiso súbito de la vía aérea y por la necesidad de contar con cuidadores y profesionales competentes para su cuidado. Los objetivos principales de este consenso son unificar criterios, promover prácticas seguras, fomentar el uso racional de los recursos y contribuir a optimizar la calidad de vida de los niños con traqueostomías y sus familias. Para poder cumplir con estos propósitos, se realizó una búsqueda exhaustiva de la literatura científica; se seleccionó, sobre la base del análisis crítico, la mejor evidencia disponible; y se formularon, por consenso interdisciplinario, una serie de recomendaciones prácticas y adaptables al contexto local.

Palabras clave: traqueostomía, traqueotomía, pediatría, manejo de la vía aérea, atención domiciliaria de salud.

\begin{abstract}
The care of the child with a tracheostomy deserves specialattention because of the potential devastating airway compromise and because of the need of competent care by caregivers and professionals. The recommendations on tracheostomy care published are few and approaches are inconsistent among different institutions. This clinical consensus statement aims to improve care for children with tracheostomies. A literature search was conducted, reviewed and revised by this group of experts, who concurred with these statements, based on the best evidence available and taking into account the local context.

Key words: tracheostomy, tracheotomy, pediatrics, airway management, home nursing.
\end{abstract}

http:/ /dx.doi.org/10.5546/aap.2016.89

\section{INTRODUCCIÓN}

La atención del niño con traqueostomía genera mucha ansiedad entre los cuidadores y los profesionales que intervienen en este proceso, ya sea en el hogar, la escuela, los centros educativos, terapéuticos o los centros de salud. Esto se debe, generalmente, al potencial riesgo para la vida que podría ocasionar el compromiso súbito de la vía aérea. Por lo tanto, mejorar las competencias de los cuidadores y profesionales vinculados en el cuidado de los niños con traqueostomía resulta primordial para mejorar la seguridad y promover la inclusión social de este grupo de niños. ${ }^{1-4}$

La traqueostomía es un procedimiento muy antiguo ${ }^{5}$ que consiste en abocar la tráquea a la piel de manera de asegurar la vía aérea y su abertura se sostiene mediante una cánula de traqueostomía. En las últimas décadas, como contracara de los avances en los cuidados críticos pediátricos y neonatales, el número de niños que ha requerido una traqueostomía se ha incrementado. ${ }^{6-14}$ Sin embargo, existen pocas publicaciones que respalden con evidencia fuerte las recomendaciones sobre su cuidado en la población infantil y ningún consenso publicado sobre su cuidado a nivel nacional.

Este primer consenso nacional sobre el cuidado del niño con traqueostomía fue pensado como una herramienta facilitadora para su manejo. Integra, en una serie de recomendaciones prácticas, la revisión 
de la mejor evidencia científica disponible con la experiencia acumulada por los expertos locales en las distintas áreas. Sus principales objetivos están dirigidos a unificar criterios, disminuir las complicaciones asociadas, promover la formación de los equipos de salud pediátricos, fomentar el uso racional de los recursos y contribuir a optimizar la calidad de vida de los niños con traqueostomía y sus familias.

Se encuentran fuera del alcance de este consenso la discusión acerca de las indicaciones para efectuar una traqueostomía, las técnicas utilizadas para su realización, las recomendaciones sobre el cuidado posquirúrgico y las recomendaciones para el cuidado del niño con traqueostomía con asistencia respiratoria mecánica prolongada.

\section{METODOLOGÍA}

Para la elaboración de este consenso, se conformó un panel de 19 expertos integrado por profesionales del Grupo de Trabajo en Discapacidad de la Sociedad Argentina de Pediatría (SAP), el Comité Nacional de Neumonología de la SAP, el Grupo de Trabajo en Kinesiología de la SAP, el Grupo de Trabajo en Enfermería Pediátrica de la SAP, el Servicio de Endoscopía del Hospital de Niños Ricardo Gutiérrez y el Servicio de Endoscopía del Hospital Nacional de Pediatría "Prof. Dr. J. P. Garrahan".

Se realizó una búsqueda biliográfica en Medline/PubMed, Cochrane Library y Lilacs, así como en bases de datos de Guías de Práctica Clínica. Se utilizó también bibliografía escrita recomendada por los expertos. La estrategia de búsqueda utilizó los encabezados de temas médicos (Medical Subject Headings; MeSH, por sus siglas en inglés) tracheostomy, tracheotomy, pediatrics, airway management, home nursing; sus descriptores en español: traqueostomía, traqueotomía, pediatría, manejo de la vía aérea y atención domiciliaria; y abarcó publicaciones en castellano e inglés de los últimos veinte años. La búsqueda se redujo de 610 a 40 publicaciones luego de descartar las duplicaciones y los artículos obviamente no relacionados. Se seleccionó, de acuerdo con el análisis crítico, la mejor evidencia disponible y se distribuyó entre el grupo de expertos para su análisis. La calidad de la evidencia recabada fue regular según el sistema GRADE (Grading of Recommendations Assessment, Development and Evaluation). ${ }^{15}$

Las recomendaciones surgidas del análisis de la evidencia científica analizada ${ }^{16-25}$ fueron sometidas a una revisión por expertos para ajustarlas a la experiencia interdisciplinaria en la práctica asistencial local.

\section{RECOMENDACIONES}

Selección del tipo de cánula de traqueostomía

Los niños pueden requerir la utilización de una cánula de traqueostomía en forma temporaria o definitiva de acuerdo con el diagnóstico de base y con el pronóstico clínico. Existen de diferentes materiales, tamaños y marcas disponibles en el mercado local para su utilización en niños. ${ }^{26}$ La selección del tipo y del tamaño de la cánula que el niño utilizará dependerá de la edad, del diagnóstico que motive la realización del procedimiento y del estado clínico al momento de su evaluación.

La edad no debe ser el único parámetro para tener en cuenta por los profesionales intervinientes en la elección del tipo de cánula de traqueostomía. Esta debe adaptarse a la vía aérea (tamaño y forma de la tráquea) y a las necesidades funcionales del niño.

Las cánulas más utilizadas y recomendadas en nuestro medio son las plásticas, de las cuales existen diferentes marcas disponibles en el mercado local. Las partes de una cánula se describen en la Figura 1. El conector universal

Figura 1. Cánulas de traqueostomía con y sin balón

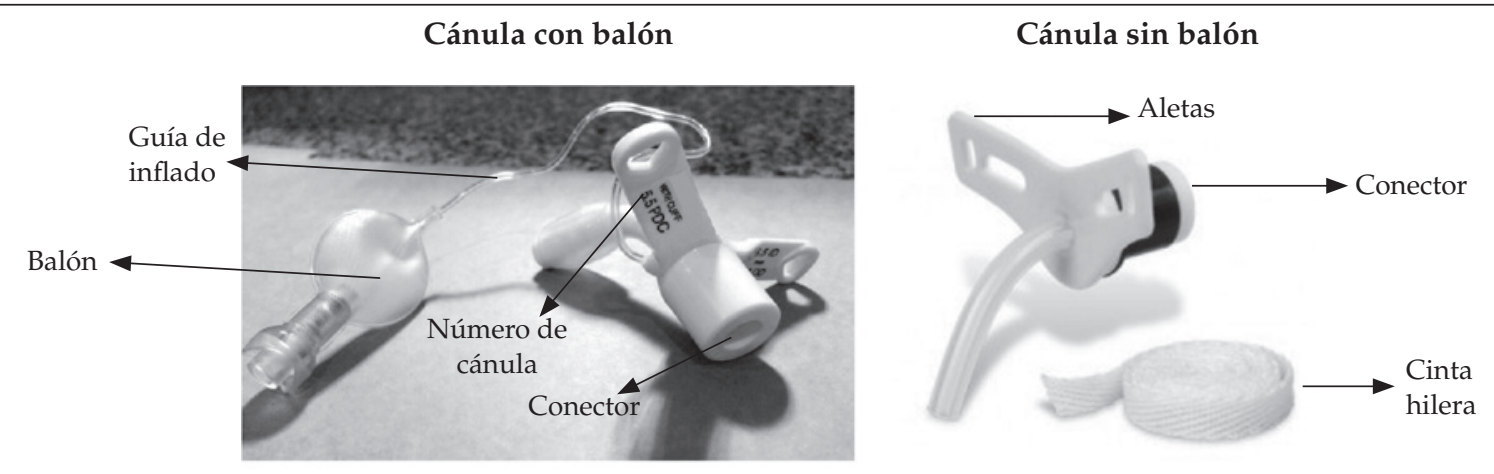


de $15 \mathrm{~mm}$ sirve para su adaptación a cualquier válvula fonatoria, tubo en " $\mathrm{T}$ ", bolsa de reanimación o circuito de ventilación mecánica.

En cuanto al tamaño, hay disponibles cánulas de traqueostomía neonatales y pediátricas para su uso en niños. Ambas difieren entre sí en su longitud. El número de la cánula se refiere a su diámetro interno, expresado en milímetros. Por ejemplo, una cánula número 4 de cualquier marca tiene un diámetro interno de $4 \mathrm{~mm}$.

En la mayoría de los casos, las necesidades de los niños se resuelven con las cánulas de traqueostomía disponibles en el mercado, y es excepcional el uso de cánulas especialmente diseñadas.

En pediatría, la indicación de las cánulas con balón es limitada. Se reserva, en general, para niños con asistencia ventilatoria mecánica que no pueden ser ventilados correctamente con cánulas sin balón. También podría considerarse su uso en los casos de niños con trastornos deglutorios graves y complicaciones clínicas.

Cuando se utiliza una cánula de traqueostomía con balón, se recomienda controlar diariamente la presión de inflado para evitar lesiones en el epitelio traqueal. La presión del balón deberá adecuarse a las necesidades funcionales del niño, sin sobrepasar los $20 \mathrm{cmH}_{2} \mathrm{O}$.

\section{Cuidado y recambio de la cánula de traqueostomía}

Se recomienda que la cánula de traqueostomía sea recambiada, al menos, una vez por semana o antes en caso de que sea necesario. Es posible que un niño requiera varios cambios en el día o en el transcurso de la semana.

Las cánulas de traqueostomía pueden ser higienizadas y reutilizadas. La higiene puede realizarse con detergente enzimático, detergente biodegradable de uso familiar o jabón líquido. El objetivo es descontaminarla, para lo que es innecesario esterilizarla, salvo que vaya a ser utilizada con otro niño. El lavado debe realizarse con agua fría. Se desaconseja el uso de clorhexidina o iodopovidona para su descontaminación. Una vez lavada, la cánula debe secarse y guardarse en un lugar limpio y seco, lista para el siguiente uso.

La vida media de la cánula dependerá del material, la técnica de cuidado, la utilización $\mathrm{y}$, por lo tanto, debe ser evaluada en forma individualizada. Con el uso, las cánulas pueden tornarse rígidas o fisurarse $y$, en ese caso, deben descartarse.
Para realizar el recambio de la cánula, se recomienda seguir los siguientes pasos:

- Realizar el lavado o higiene de manos.

- Colocar al niño en decúbito dorsal con realce debajo de los hombros.

- Aspirar las secreciones.

- Higienizar la piel periostomal con agua y jabón y desatar la sujeción.

- Realizar maniobra de hiperextensión del cuello o apertura del ostoma con los dedos.

- Extraer la cánula en uso y colocar una cánula limpia.

- Fijar la cánula utilizando las aletas con un método de sujeción seguro.

La posición para realizar el recambio podría ser otra. Se debe buscar aquella en la que el niño se encuentre más confortable, dependiendo, a su vez, de la edad y de la habilidad del niño para recambiarse la cánula él mismo.

Se recomienda utilizar la técnica limpia para el recambio de la cánula en el hogar o la comunidad (cánula limpia y manos limpias). Se sugiere la técnica limpia modificada para el recambio en instituciones o cuando es llevado a cabo por cuidadores ajenos a la familia (cánula limpia y manoplas) (Figura 2). Se reserva la utilización de la técnica estéril para el cuidado en las unidades de terapia intensiva.

Se recomienda la utilización de lubricantes hidrosolubles, como solución fisiológica o lidocaína jalea, para facilitar la inserción de la cánula limpia. Se desaconseja el uso de vaselina como lubricante.

Existen distintos métodos de sujeción de la cánula de traqueostomía. No hay evidencia que asocie la utilización de collarines comerciales a una menor incidencia de complicaciones. Además, en ocasiones, el material velcro se desgasta o es desabrochado por el niño cuando se mueve. La cinta hilera anudada y los collarines fabricados en forma casera con velcro o cinta hilera son una alternativa aceptable. Se recomienda evitar la utilización de cintas de resistencia dudosa, como barbijos.

Debe prestarse particular atención al nudo, cualquiera sea el método de sujeción elegido, que debe ser seguro y firme. La sujeción debe permitir que pase un dedo entre el collarín y la piel. Debe ser lo suficientemente firme como para evitar la decanulación accidental, pero se debe evitar que la tensión sea excesiva como para generar lesiones cutáneas u oclusión vascular.

El collarín debe permanecer limpio y seco. No hay una frecuencia fija recomendada para 
su recambio y debe ser evaluado en forma individualizada. Se recomienda que la piel periostomal permanezca limpia y seca. Se desaconseja el uso de talcos en la piel cercana a la ostomía.

\section{Aspiración}

Mantener la vía aérea permeable mediante la aspiración de las secreciones traqueales es una medida de vital importancia en el cuidado del niño con traqueostomía. . $^{27-28}$

Existen distintos sistemas de aspiración, además de los sistemas hospitalarios, que permiten la aspiración de secreciones en el hogar y en los lugares en los que se desenvuelve el niño (paseos, escuelas, centros educativos, terapéuticos, etc.), como aspiradores eléctricos, aspiradores manuales (Figura 3), aspirador tipo pipeta De Lee y aspiradores a baterías.

El objetivo de la aspiración de la cánula de traqueostomía es mantenerla permeable de la manera más efectiva y ocasionando el menor daño o malestar posible.
La aspiración con técnica de sonda medida es la técnica recomendada. Consiste en no aspirar más allá de la longitud de la cánula para evitar dañar la mucosa traqueal con las inserciones profundas.

Para realizar la aspiración de secreciones, se recomienda seguir los siguientes pasos:

- Realizar el lavado o higiene de manos.

- Introducir la sonda sin sobrepasar la longitud de la cánula.

- Retirar la sonda aspirando con un movimiento rotatorio.

La técnica de aspiración dependerá del ámbito en el que se encuentre el niño. En las unidades de cuidados críticos, se prefiere la técnica de aspiración estéril (sonda estéril y guantes estériles). En el ámbito hospitalario, fuera de las unidades críticas (cuidados intermedios, salas de pediatría) o en los centros de rehabilitación, se prefiere la técnica de aspiración limpia modificada (sonda estéril con manoplas). Finalmente, en el domicilio o la comunidad, se prefiere la técnica
Cómo realizar el recambio de la cánula
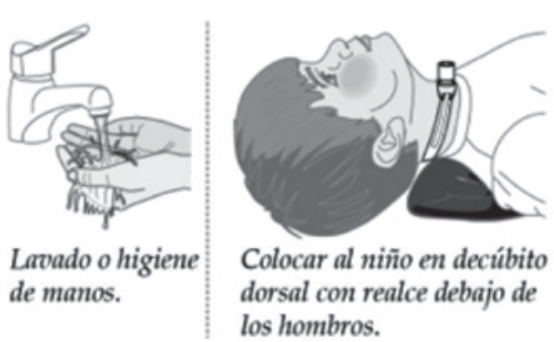
los hombros.

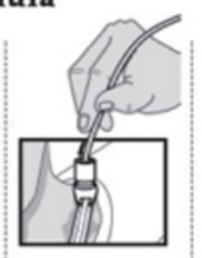

Aspirar las secreciones.

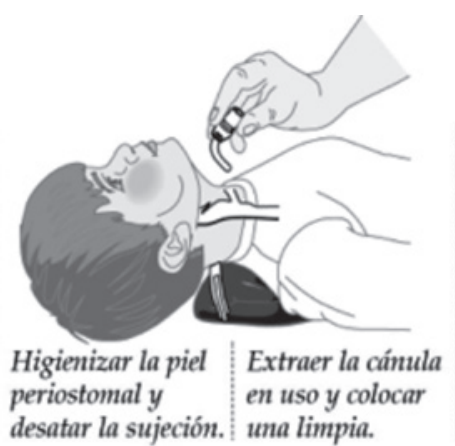

desatar la sujeción. una limpia.

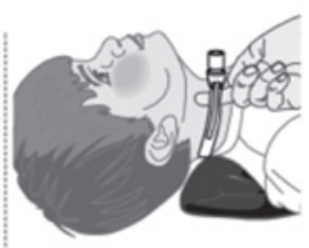

Fijar la cánula utilizando las aletas con un método de sujeción seguro.

Hospital, centros de rehabilitación: cánula limpia y manoplas

Domicilio: cánula limpia y manos limpias
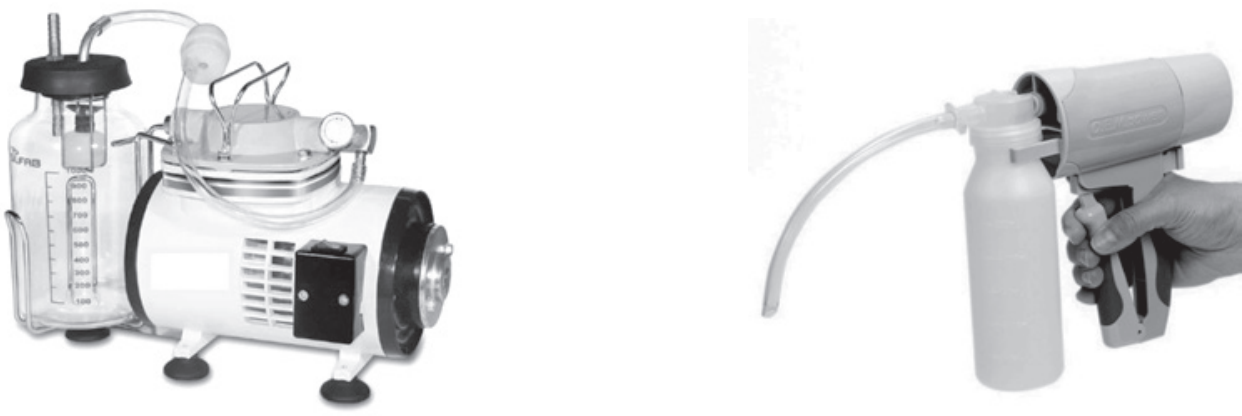
de aspiración limpia, es decir, con sonda limpia y manos limpias (Figura 4).

Se recomienda que la sonda de aspiración no ocluya completamente el pasaje de aire durante la aspiración, que la técnica sea breve (5-10 segundos) y que se retire con un movimiento rotatorio que permita el pasaje de los orificios

FIgURA 4. Técnica de aspiración de secreciones traqueales

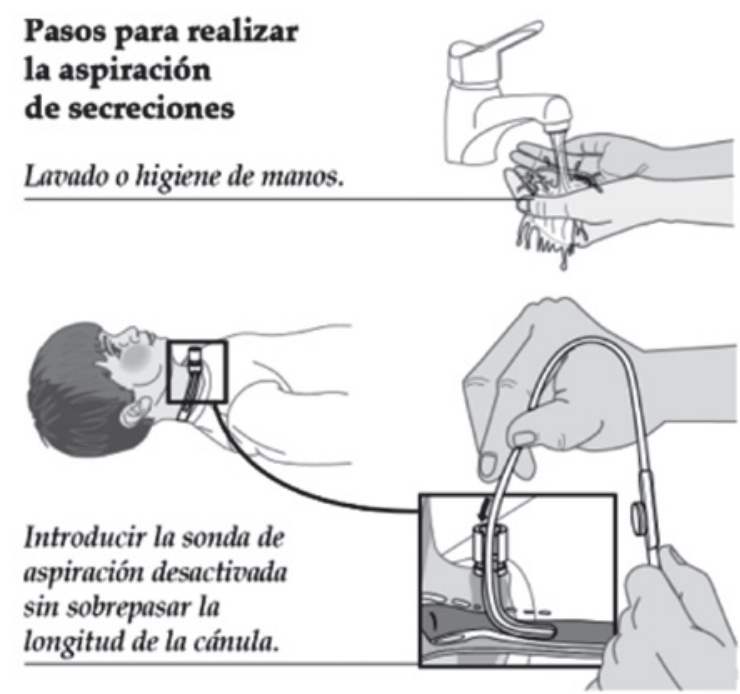

Retirar la sonda de aspiración actioada con un movimiento rotatorio durante un tiempo no superior a 10 segundos.
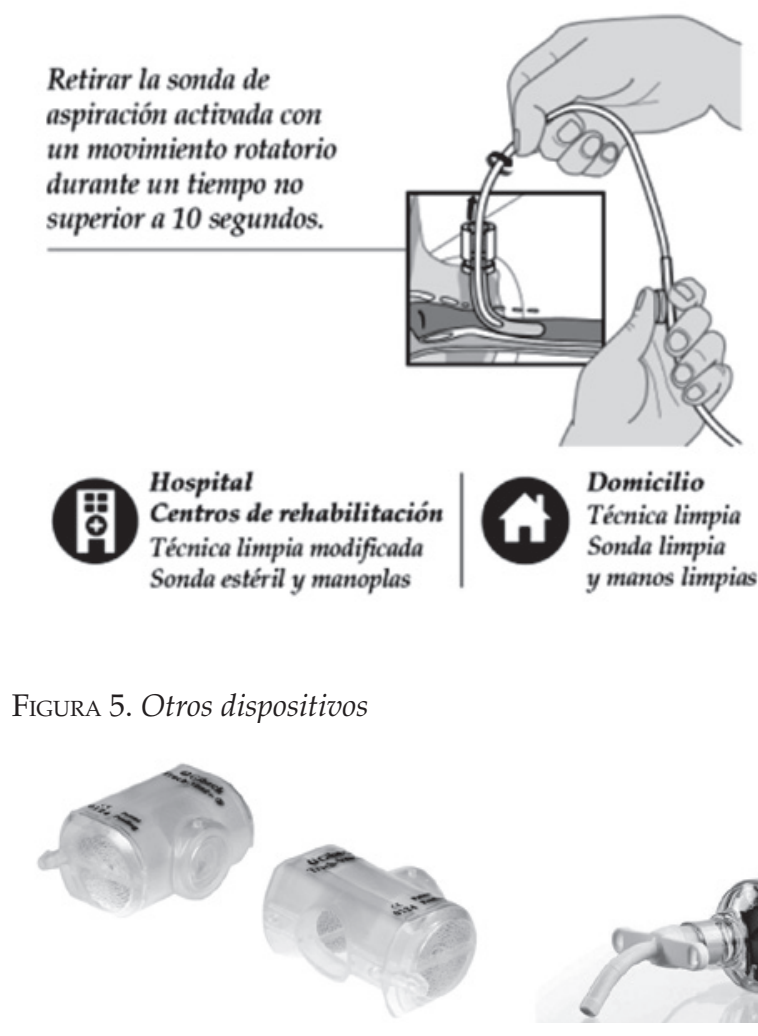

Figura 5. Otros dispositivos
Humidificadores pasivos

Humidificadores pasivos

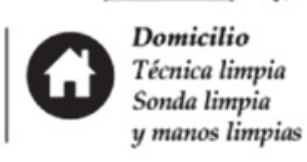

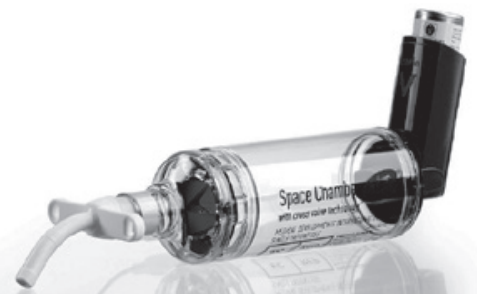

Aerocámaras de la sonda de aspiración por toda la pared de la cánula de traqueostomía.

La instilación de solución fisiológica y la realización de nebulizaciones en forma rutinaria antes de la aspiración no están recomendadas. En caso de constatar secreciones espesas, se aconseja instilar solución fisiológica antes de la aspiración y revisar el sistema de humidificación utilizado.

La frecuencia de la aspiración dependerá de la evaluación clínica y de las necesidades de cada niño. Se recomienda verificar la permeabilidad de la cánula de traqueostomía antes de la alimentación, antes de dormirse y al levantarse.

\section{Humidificación}

Normalmente, la vía aérea superior funciona como filtro, calentador y humidificador del aire inspirado. Esta función se ve alterada en el niño con traqueostomía, ya que hay déficit en la filtración y humidificación. La utilización de humidificadores pasivos (Figura 5) u otros métodos de barrera alternativos debe ser evaluada en forma individual tomando en cuenta factores como eficacia, seguridad, costo, actividad social, oxigenoterapia domiciliaria y conveniencia. ${ }^{29}$

En neonatos, lactantes hipotónicos y niños con secreciones espesas, no se recomienda la utilización de humidificadores pasivos.

\section{Administración de medicación}

En caso de ser necesaria, la administración de medicación en aerosol debe realizarse a través de una aerocámara con conector para traqueostomía (Figura 5).

También se puede utilizar medicación nebulizada por vía traqueal, pero se debe recordar que la nebulización es solo un medio para suministrar fármacos, no para humidificar las secreciones. Se sugiere no utilizar medicación instilada por vía traqueal.

\section{Fonación}

Los niños con traqueostomía tienen limitada la emisión de sonido. La comunicación oral 
puede ser facilitada, en este grupo de niños, mediante la autooclusión de la cánula o mediante la utilización de una válvula fonatoria (Figura 5). Esta consiste en una válvula unidireccional que permite el pasaje de aire por la traqueostomía durante la inspiración, pero no durante la espiración, lo que fuerza el pasaje de aire a través de la laringe. Si bien las válvulas de fonación pueden utilizarse en todas las edades, en el caso de los niños más pequeños, su uso puede verse limitado por la relación entre el diámetro de la cánula y la tráquea, que no permite un adecuado pasaje de aire translaríngeo.

Si el niño tiene una cánula con balón, debe desinflarse siempre antes de usar la válvula de fonación.

Se recomienda la evaluación laríngea mediante métodos radiológicos o endoscópicos antes de indicar el uso de válvulas fonatorias.

\section{Monitoreo}

El niño con traqueostomía puede requerir la supervisión de un cuidador entrenado para realizar las actividades acordes a su edad y a sus posibilidades hasta que adquiera autonomía y competencia para el autocuidado.

El mejor monitor del niño con traqueostomía es un cuidador bien entrenado. Por lo tanto, este debe lograr competencias para el cambio de la cánula, para identificar signos de alarma, para el manejo del material descartable y para el uso del equipamiento portátil disponible localmente para el manejo ambulatorio. ${ }^{30-36}$ Si bien no se recomienda el uso de monitores, como el oxímetro de pulso, en forma sistemática, su utilización deberá evaluarse en cada caso en particular.

\section{Complicaciones}

Las complicaciones más frecuentes, graves y evitables son la decanulación no intencional y la obstrucción. Otras complicaciones son la presencia de sangrado, granulomas o traqueítis.

Para prevenir la decanulación no intencional, se recomienda seguir la técnica de sujeción descrita y controlar periódicamente la tensión de la fijación. La decanulación se resolverá con la colocación de la cánula.

Siempre que haya una descompensación súbita del niño, debe descartarse, en primera instancia, la obstrucción de la cánula. Esta se resolverá con el recambio inmediato.

El sangrado por la cánula de traqueostomía se debe frecuentemente a traumatismos causados por la aspiración de secreciones. Por lo tanto, debe revisarse la técnica de aspiración en caso de presentarse y recomendarse la técnica de sonda medida. Si el sangrado es leve y autolimitado, no es necesario consultar al especialista.

El granuloma es una reacción inflamatoria muy frecuente en el ostoma y se debe a la presencia de un cuerpo extraño, como es la cánula de traqueostomía. El granuloma es friable y puede sangrar. Si es plano y periférico, no se debe tomar ninguna conducta. Si obstruye la traqueostomía y dificulta el recambio de la cánula, se recomienda consultar al especialista para su exéresis. La topicación de los granulomas con distintas sustancias no está recomendada.

La traqueostomía genera una abertura de la vía aérea, que facilita su contaminación. El lavado de manos es, por lo tanto, la medida más efectiva para la prevención de infecciones. En caso de traqueítis, el cultivo rutinario de las prótesis o de las secreciones traqueales no está recomendado. En caso de infecciones de la vía aérea, el método de elección para el rescate bacteriano es el lavado bronquioloalveolar.

Otra complicación posible es la dificultad en la recanulación. En este caso, se recomienda revisar la técnica de recambio. Si persiste la dificultad, se debe utilizar una cánula de traqueostomía enhebrada con un tubo endotraqueal que sobrepase 1-2 cm la longitud de la cánula. El tubo endotraqueal biselado facilita su inserción en la tráquea. Si aun así, no puede hacerse, se deberá colocar una cánula de menor tamaño o el tubo endotraqueal a través del ostoma para la estabilización del niño.

\section{Inclusión social}

En el marco de la Convención de los Derechos de las Personas con Discapacidad, ${ }^{37}$ se debe promover el acceso de los niños con traqueostomía a la enseñanza obligatoria, ${ }^{38-40}$ a las actividades recreativas y a la rehabilitación, considerando los ajustes razonables que se requieran en función de las necesidades individuales.

La posibilidad de aprender habilidades para la vida diaria propiciará la participación plena de los niños en la sociedad, en igualdad de condiciones, como miembros de la comunidad y con miras a desarrollar plenamente su potencial.

\section{DISCUSIÓN}

Para la elaboración de este consenso, se ha decidido considerar la mejor evidencia disponible, revisada por expertos locales en distintas disciplinas y complementada, en 
aquellos casos en los que existió controversia o insuficiente evidencia, con su experiencia, hasta tanto se dispusiera de información adecuadamente sustentada. Por lo tanto, el profesional o interviniente en el cuidado del niño con traqueostomía puede utilizar criterios distintos a los de este consenso para la toma de decisiones basados en consideraciones de riesgos, disponibilidad, costos o preferencias de los usuarios.

\section{REFERENCIAS}

1. Cameron TS, McKinstry A, Burt SK, Howard ME, et al. Outcomes of patients with spinal cord injury before and after introduction of an interdisciplinary tracheostomy team. Crit Care Resusc 2009;11(1):14-9.

2. Cetto R, Arora A, Hettige $\mathrm{R}, \mathrm{Nel} \mathrm{M}$, et al. Improving tracheostomycare:aprospectivestudyof themultidisciplinary approach. Clin Otolaryngol 2011;36(5):482-8.

3. McGrath BA, Calder N, Laha S, Perks A, et al. Reduction in harm from tracheostomy-related patient safety incidents following introduction of the National Tracheostomy Safety Project: our experience from two hundred and eighty-seven incidents. Clin Otolaryngol 2013;38(6):541-5.

4. McGrath BA, Bates L, Atkinson D, Moore JA. Multidisciplinary guidelines for the management of tracheostomy and laryngectomy airway emergencies. Anaesthesia 2012;67(9):1025-41.

5. Szmuk P, Ezri T, Evron S, Roth Y, et al. A brief history of tracheostomy and tracheal intubation, from the Bronze Age to the Space Age. Intensive Care Med 2008;34(2):222-8.

6. Carron JD, Derkay CS, Strope GL, Nosonchuk JE, et al. Pediatric tracheotomies: changing indications and outcomes. Laryngoscope 2000;110(7):1099-104.

7. Estournet-Mathiaud B. Tracheostomy in chronic lung disease: care and follow-up. Pediatr Pulmonol 2001;(Suppl 23):135-6.

8. Davis GM. Tracheostomy in children. Paediatr Respir Rev 2006;7(Suppl 1):S206-9.

9. Mahadevan M, Barber C, Salkeld L, Douglas G, et al. Pediatric tracheotomy: 17 year review. Int J Pediatr Otorhinolaryngol 2007;71(12):1829-35.

10. Graf JM, Montagnino BA, Hueckel R, McPherson ML. Pediatric tracheostomies: a recent experience from one academic center. Pediatr Crit Care Med 2008;9(1):96-100.

11. Fraga JC, Souza JC, KruelJ. Pediatric tracheostomy. JPediatr (Rio) 2009;85(2):97-103.

12. DeutschES. Tracheostomy:pediatric considerations. Respir Care 2010;55(8):1082-90.

13. Spentzas T, Auth M, Hess P, Minarik M, et al. Natural course following pediatric tracheostomy. I Intensive Care Med 2010;25(1):39-45.

14. Gallagher TQ, Hartnick CJ. Pediatric tracheotomy. Adv Otorhinolaryngol 2012;73:26-30.

15. Guyatt GH, Oxman AD, Vist G, Kunz R, et al. GRADE: an emerging consensus on rating quality of evidence and strength of recommendations. BMJ 2008;336(7650):924-6.

16. Fitton CM. Nursing management of the child with a tracheotomy. Pediatr Clin North Am 1994;41(3):513-23.

17. Sherman JM, Davis S, Albamonte-Petrick S, Chatburn RL, et al. Care of the child with a chronic tracheostomy. Am J Respir Crit Care Med 2000;161(1):297-308.

18. Lewarski JS. Long-term care of the patient with a tracheostomy. Respir Care 2005;50(4):534-7.
19. Wilson M. Tracheostomy management. Paediatr Nurs 2005;17(3):38-43.

20. Dhand R, Johnson JC. Care of the chronic tracheostomy. Respir Care 2006;51(9):984-1001.

21. Eber E, Oberwaldner B. Tracheostomy care in the hospital. Paediatr Respir Rev 2006;7(3):175-84.

22. Cincinnati Children's Hospital Medical Center. Best evidence statement (BESt). Basic pediatric tracheostomy care. Rockville: Agency for Healthcare Research and Quality. 2011. [Acceso: 3 de agosto de 2015]. Disponible en: http:/ / www.guideline.gov/ content.aspx?id=34159.

23. Hettige R, Arora A, Roberson DW, Narula AA. Recent developments to improve the standards of tracheostomy care. British Journal of Intensive Care 2013;23(3):89-92.

24. Mitchell RB, Hussey HM, Setzen G, Jacobs IN, etal. Clinical consensus statement: tracheostomy care. Otolaryngol Head Neck Surg 2013;148(1):6-20.

25. Paz F, Zamorano A, Paiva R, Hernández $\mathrm{Y}$, et al. Cuidados de niños con traqueostomía. Neumol Pediatr 2008;3(Supl):64-70.

26. Salas Campos L, Solé i Fábregas A, Rodríguez Hebra I, Martín Rivero B, et al. Cánulas de traqueostomía. Rev Enferm 1998;21(238):95-101.

27. American Association for Respiratory Care. AARCClinical Practice Guideline: Suctioning of the patient in the home. Respir Care 1999;44(1):99-104.

28. Ireton J. Tracheostomy suction: a protocol for practice. Paediatr Nurs 2007;19(10):14-8.

29. McNamara DG, Asher MI, Rubin BK, Stewart A, et al. Heated humidification improves clinical outcomes, compared to a heat and moisture exchanger in children with tracheostomies. Respir Care 2014;59(1):46-53.

28. Fiske E. Effective strategies to prepare infants and families for home tracheostomy care. Adv Neonatal Care 2004;4(1):42-53.

29. Botto H, Nieto M, Zanetta A, Rodríguez H. Manejo domiciliario del niño traqueotomizado. Arch Argent Pediatr 2008;106(4):351-5.

30. Caussade S, Paz F, Ramírez M, Navarro H, et al. Experiencia clínica en el manejo domiciliario de niños traqueostomizados. Rev Med Chile 2000;128(11):1221-6.

31. Oberwaldner B, Eber E. Tracheostomy care in the home. Paediatr Respir Rev 2006;7(3):185-90.

32. Flynn AP, Carter B, Bray L, Donne AJ. Parents' experiences and views of caring for a child with a tracheostomy: a literature review. Int J Pediatr Otorhinolaryngol 2013;77(10):1630-4.

33. Joseph RA. Tracheostomy in infants: parent education for home care. Neonatal Netw 2011;30(4):231-42.

34. Norwood MG, Spiers P, Bailiss J, Sayers RD. Evaluation of the role of a specialist tracheostomy service. From critical care to outreach and beyond. Postgrad Med J 2004;80(946):478-80.

35. Naciones Unidas. Convención sobre los Derechos de las Personas con Discapacidad y Protocolo Facultativo. 2006. [Acceso: 3 de agosto de 2015]. Disponible en: http:// www.un.org/disabilities/documents/convention/ convoptprot-s.pdf.

36. Porter SM, Page DR, Somppi C. Emergency preparedness in the school setting for the child assisted by medical technology. Tracheostomies, ventilators, and oxygen. NASN Sch Nurse 2013;28(6):298-305.

37. Spratling R, Minick $P$, Carmon M. The experiences of school-age children with a tracheostomy. J Pediatr Health Care 2012;26(2):118-25.

38. PatelMR,ZdanskiCJ,AbodeKA, ReillyCA, etal. Experience of the school-aged child with tracheostomy. Int J Pediatr Otorhinolaryngol 2009;73(7):975-80. 